Research Article

\title{
A Cross-Border E-Commerce Approach Based on Blockchain Technology
}

\author{
Zhao Hongmei (iD \\ Hunan Sany Polytechnic College, Hunan, Changsha 412006, China \\ Correspondence should be addressed to Zhao Hongmei; t20071490@csuft.edu.cn
}

Received 23 June 2021; Accepted 6 July 2021; Published 16 July 2021

Academic Editor: Muhammad Usman

Copyright (c) 2021 Zhao Hongmei. This is an open access article distributed under the Creative Commons Attribution License, which permits unrestricted use, distribution, and reproduction in any medium, provided the original work is properly cited.

In the current cross-border electronic commerce (e-commerce) system, various document recording and authorization processes are cumbersome, record sharing efficiency is low, and identity verification is difficult. A method of asymmetric encryption technology combining Blockchain technology and cryptography is proposed. The advantages of asymmetric encrypted communications include high security and ease of multiparties communication collaboration, being applied to a peer-to-peer network formed by Blockchain technology, and making cross-border e-commerce record cross-domain sharing traceable, data immutable, and identity verification simplified. First of all, based on the immutable modification of Blockchain technology and asymmetric encryption technology, file synchronization contracts and authorization contracts are designed. Its distributed storage advantages ensure the privacy of users' cross-border e-commerce information. Second, the design of the cross-domain acquisition contract can effectively verify the identity and transmission efficiency of both parties to the data sharing, so that illegal users can be safely filtered without a third-party notary institution. The simulation experiment results show that the solution proposed in this paper has obvious advantages in data antitheft, multiparty authentication, and saving system overhead compared with traditional cloud computing methods to solve the problem of sharing medical records. It provides a reference for solving the security problems in the process of data sharing by using the advantages of Blockchain's decentralization and auditability and provides reference ideas for solving the problems of data sharing and cross-domain authentication.

\section{Introduction}

In recent years, with the rapid development of China's local e-commerce and the continuous improvement of its business model, it has not only stepped into the forefront of the world but also brought unprecedented opportunities for the development of China's cross-border e-commerce. Therefore, it is imperative to build a balanced and sustainable cross-border e-commerce system. But at present, the traditional e-commerce model, especially the centralized e-commerce model, cannot meet the sustainable development of cross-border e-commerce. Especially in the aspects of traceability, collaboration, payment, and contract, they are facing great challenges. At the same time, the emergence of digital currency represented by bitcoin, especially the underlying technology bitcoin relies on Blockchain technology, has aroused widespread concern in academic circles.
In 2016, Blockchain technology has developed rapidly in the world, so it is also known as the first year of Blockchain application. Blockchain technology is considered to be another subversive technological innovation after Internet technology, and it is also permeating into cross-border e-commerce, network financial services, the Internet of things, and other fields. Its rapid development momentum is expected to lead the transformation of the Internet economy mode from application-driven innovation mode to technology-driven innovation mode. One belt, one road, and the other cross-border electric business, which are advocated by China, will also provide new directions for the development of cross-border electricity providers.

Cross-border e-commerce is a kind of business activity, which takes the transactions belonging to different customs as the main body and makes the two sides reach a certain agreement through the e-commerce platform, to carry out 
payment and settlement and transport goods across the border, to complete the transaction. In recent years, as the growth rate of the global economy and trade gradually tends to be flat, the circulation of goods is slow. To solve this problem, we should constantly accelerate the circulation of goods, open up overseas markets, and improve the growth rate of the economy and trade. More and more enterprises to shorten the distance with foreign consumers accelerate commodity sales. In this economic environment, crossborder e-commerce should be shipped out; now crossborder e-commerce is still developing rapidly, gradually affecting the world's economic trend. The emergence of cross-border e-commerce greatly reduces the transaction cost between e-commerce enterprises and foreign customers, simplifies the transaction process, directly and effectively speeds up the capital turnover, and provides an efficient cross-border trade channel for the world.

In the process of cross-border transactions, the credit rating of cross-border e-commerce enterprises is different due to the differences in economy, culture, and system between different countries. At present, there is no open enterprise credit sharing platform between countries, which makes it difficult for enterprises of different countries to evaluate each other's credit effectively in the process of crossborder transactions, which makes it difficult for enterprises of both sides of the transaction to trust each other, so that enterprises have adverse selection consequences, and then affect the overall development of cross-border e-commerce.

It has realized the traceability function of logistics, but it is not satisfactory in cross-border e-commerce logistics. The driving force of cross-border trade is to export products with comparative advantages to other countries. For example, oil in the Middle East, machinery products in Europe, food in Central America, and manufacturing in Asia all have their comparative advantages. However, the asymmetric information of the importing and exporting countries often leads to adverse selection in the transaction of such products. Even more, some domestic illegal businesses turn domestic fake and shoddy goods into imported goods by counterfeiting logistics information. These not only damage the fundamental interests of consumers but also harm the development of cross-border e-commerce and even lead to international disputes.

Due to the geographical location of cross-border e-commerce and the differences in foreign exchange control policies of different countries, the current cross-border payment means generally cannot achieve real-time arrival, and the rapid change of exchange rate increases the risk of exchange rate fluctuation caused by the enterprise's foreign exchange exposure in the payment process to a certain extent. Besides, today's cross-border e-commerce transactions are generally conducted through the cross-border e-commerce platform and all transaction data is also stored on the virtual platform. Therefore, the security risks in the payment process cannot be completely avoided. Under the current cross-border e-commerce system, the cross-border payment platform cannot effectively restrict the cross-border e-commerce platform. At the same time, the inconsistency of financial policies and regulations in the world also makes it difficult to effectively supervise cross-border payment. This lack of supervision needs the mode of enterprise self-discipline, which objectively increases the credit risk and transaction cost of enterprises in the transaction process.

Because cross-border e-commerce is the trade between countries behind individual and enterprise transactions, the complexity of cross-border e-commerce is much higher than that of domestic e-commerce. Due to the need for the participation of customs, inspection and quarantine, logistics, and other subjects, it also needs to spend a lot of manpower and material costs in the aspects of the review, reconciliation, customs clearance, and so forth. Therefore, enterprises engaged in cross-border e-commerce not only need to deal with cross-border logistics, exchange rate, and other uncertain factors but also need to get through many links, thus pushing up the economic cost and time cost.

The rest of this paper is organized as follows. In Section 2 , related work is presented followed by the system model and proposed work in Section 3. In Section 4, performance analysis is presented followed by experimental evaluation in Section 5. Finally, the paper is concluded in Section 6.

\section{Related Work}

Satoshi Nakamoto introduced a fully decentralized digital currency exchange system based on distributed systems and cryptography in 2008 [1]. This is the first time Blockchain technology has been used. Blockchain demonstrated the ability to execute peer-to-peer digital currency payments in an untrustworthy setting. In [2], the author launched the Ethereum plan, which enables people to create transactions to fulfill any system transfer function and automate processes by constructing a Blockchain framework in Turing's full language. In $[3,4]$, Melanie Swan first released the block exchange innovation trading platform, which was a significant landmark in the modern financial market's decentralization development. The British government published a report on Blockchain in [5]. "Distributed Ledger Technology: Beyond Block Chain" is a research study that advocates the use of Blockchain for financial and government affairs. The People's Bank of China held a digital currency workshop to look into the possibility of using Blockchain technology to offer digital currency in order to increase the quality, convenience, and transparency of the financial transactions. In [6], the authors explain the Blockchain in terms of data design, encryption properties, and network structure. The blockchain is cryptographically promised, and it is not changeable and emissions reductions decentralized ledger, a data structure composed of a relational database, and a data structure composed of a relational database. In [7], the authors developed Blockchain research from 2014 to 2018, claiming that Blockchain work is still in its infancy in terms of theoretical context, methodological heterogeneity, and empirically based study. According to the authors in [8], Blockchain innovations have the ability to address economic inequality by overcoming the 4 times more likely of geographical exposure, high costs, inadequate banking goods, and economic ignorance. The authors in [9] presented multiple research studies and discussed the effect of 
Blockchain on supply chain management. Reference [10] showed early signs of Blockchain being used in supply chain operations to improve liability and clearness. This investigation shows how Blockchain can help supply chain managers achieve their goals through a range of techniques. The Blockchain is used to solve the quality control issue in the agriculture supply chain in [11]. In [12], the authors addressed the market continuity equation of the supply chain quality control issue. The authors in [13] looked into the actions of individual consumers when it came to the use of Blockchain in the supply chain. Life span of results, social impact, enabling conditions, openness, and confidence have all been shown to have varying levels of control on users' actions intentions against Blockchain. The authors in [14] gathered empirical evidence and concluded that intense competition, sophistication, expense, and comparative have major effects on Blockchain adoption. The rest of the work is organized as follows: in Section 3, the proposed model for cross-border e-commerce approach based on Blockchain technology is explained; in Section 4, performance evaluation is discussed; in Section 5, the experimental work is explained; and in Section 6, the conclusion is explained.

\section{System Model}

The block diagram of our proposed cross-border e-commerce approach based on Blockchain technology is presented in Figure 1, wherein the users first register themselves in a hospital of a particular country. After users' (patient and doctor) registration, they verify themselves by requesting to EHRs manager; else, a penalty is generated on this specific ID. Following effective authentication, the approved user can now upload/download healthcare data, which can then be stored on the Blockchain after concerned patient and practitioner approval. This approved data become a transaction and store in the Interplanetary File System (IPFS) by calculating the desired hash; this IPFS is a distributed storage mostly used in Blockchain technology. After storage, the block is stored in the transaction pool which consists of two types of transaction, that is, the transactions received from the Blockchain and the transaction that need to be store on the Blockchain. After successful mining, the block is added to the Blockchain network by the approval of the Policy List, that is, checking the rules/regulations for different countries in order to interact with the Blockchain [15-20].

The block structure of the proposed system is presented in Figure 2; it includes Hash of previous block, Nonce, Time Stamp, Version, and Markel Root (consists of hash of the transactions); the block calculates its own hash, which can be given to other blocks as its previous block hash.

3.1. Application Scenarios. Traditional cross-border e-commerce record solutions are limited by the efficiency of each platform in terms of storage and real-time viewing of transaction records. For cross-border e-commerce merchants, this will be a great time overhead. In addition, once a platform loses this transaction record, it cannot provide a complete record in the subsequent e-commerce transaction process, so that it may not be able to provide a credible record in cross-border aspects. Due to the increasing development of the current network, the evaluation of e-commerce transactions requires multiple inspections, and sometimes even more than 10 items are inspected. Effective and complete preservation of these transaction records is essential. For cross-border e-commerce transaction records, the transaction records often store the private information of the transaction personnel. Our work is how to ensure that the transaction information stored in the database is not obtained by criminals. To solve the above problems, this solution addresses the four core issues currently in the field of transaction records: (1) how to store e-commerce transaction records safely and effectively, (2) how to upload transaction records in real time, (3) how to set access control to filter illegal persons, and (4) how to conveniently download transaction records across domains. Design three Ethereum smart contracts based on Ethereum: file synchronization contract, authorization contract, and crossdomain acquisition contract.

3.2. Smart Contract Algorithm. This algorithm can be explained in the following sections.

3.2.1. File Synchronization Contract. To ensure the files uploaded to RSME are safe, we use an asymmetric encryption algorithm to encrypt the user in the encryption layer. In the platform of this scheme, we use three encryption algorithms to save the digital password: hash algorithm, ECC algorithm, and transaction record algorithm. The hash algorithm SHA-256 encrypts the user ID, ECC encrypts the public-private key pair, and the transaction record algorithm is responsible for recording the intermediate events in the folder FOLDAADDRESS [21-24]. We use the IPNs protocol to hash and encrypt the folder. The early worker information is represented by the following:

$$
\begin{aligned}
& \text { merchants }\{\text { ID (Identity), PK (Public - key), } \\
& \text { SK (Private - key), Folder - Address, Permission }\} .
\end{aligned}
$$

The user's unique ID is used to uniquely identify the e-commerce identity after SHA-256 hash algorithm was used to encrypt the data. The ECC algorithm encrypts the SK (private key) pair, PK (public key) is used to encrypt the transaction of the e-commerce, and a single file ID, that is, HashID, is gained after encryption. It can be expressed by the following formula [25-29]:

$$
\begin{gathered}
\text { SHA - 256 (PK (Public Key), EMR) } \\
=\text { HashID (Hash value of ID). }
\end{gathered}
$$

The encryption and synchronization procedures can be seen in Figure 3.

The e-commerce company itself holds a public key and private key pairs, i.e., PK and SK. Afterwards, the platform transaction is finalized and the PK is assigned to platform A. The platform uses formula (2) to encrypt the e-commerce transaction record file to gain the desired encrypted file, i.e., 


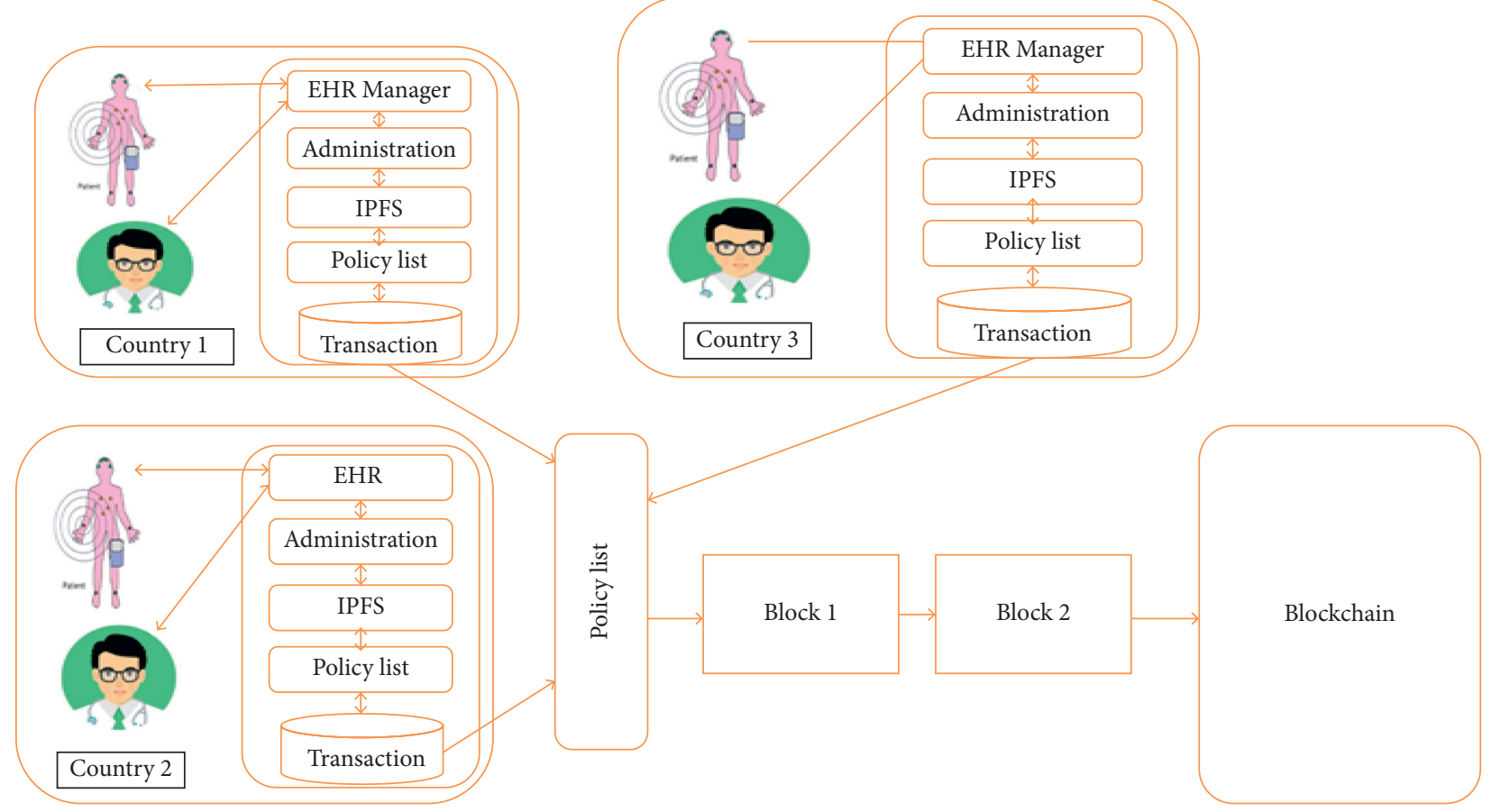

Figure 1: Network model of the proposed cross-border e-commerce approach based on Blockchain technology.

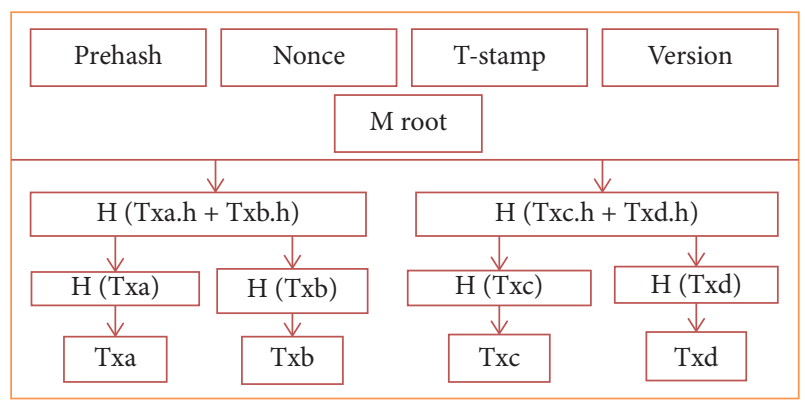

FIGURE 2: Block structure of the proposed cross-border e-commerce approach based on Blockchain technology.

SecFile, and its record file is kept in the file address of the transaction record folder for uploading to the IPFS service [30-36].

The user's authentication protocol is explained in Algorithm 1. First, the smart device (SD) encodes the requested user ID using the EHR Manager's public key and assigns it to an encrypted message, which is then transmit to the EHR Manager to decrypt it using the EHR Manager's private key. The ID is then sent to the Admin Unit, where it can be reviewed under Policy in the Policy List; if it appears there, authentication is successful; else, a penalty is applied to this ID, which will serve as a message of warning to the recipient in our scheme.

The proposed system's algorithm (Algorithm 2) describes how to add a fresh block to the Blockchain. To begin, a list of possible miners is given by the miner, who is bound by the Policy List's clear limit. The block header is then computed. The targeted hash along with nonce is determined if the target becomes accurate. If the chosen miner is validated and all of the situations are met, the block is implemented; else, the block is rejected.

The entire encryption process is given in Algorithm 3.

3.2.2. License Agreement. In this section, we mainly describe the file transfer process, which is mainly described by the automatically executed intelligent contract file and transmits the Blockchain in the Ethernet. This process is as follows.

First, the layer encrypted by the hash algorithm is to output the unique file ID and encryption security file securityfile. Secondly, when the intelligent contract receives the encrypted ciphertext file securityfile, these files will be automatically transferred to the private protocol network, and before the file is uploaded, the private protocol will automatically check if these files are copied, so the purpose is to save bandwidth. When all difficulties are successful, we have a unique file ID through formula (3), but these records usually include the following types $[37,38]$ :

\section{SHA 256\{PK, RecordFile}

(Pic1\&Picn, Video1\&Videon, Text1\&Textn) $\}=$ HashID1.

A folder value of hash obtained by encryption comprises many files, which are represented by the following symbols in this article:

(i) $\mathrm{Pic}_{11}, \ldots, \mathrm{Pic}_{1 n}$

(ii) Video $_{11}, \ldots$, Video $_{1 n}$

(iii) $\operatorname{Text}_{11}, \ldots, \mathrm{Text}_{1 n}$

In the process of transaction, if a company has transaction records on multiple platforms, then, in these private 


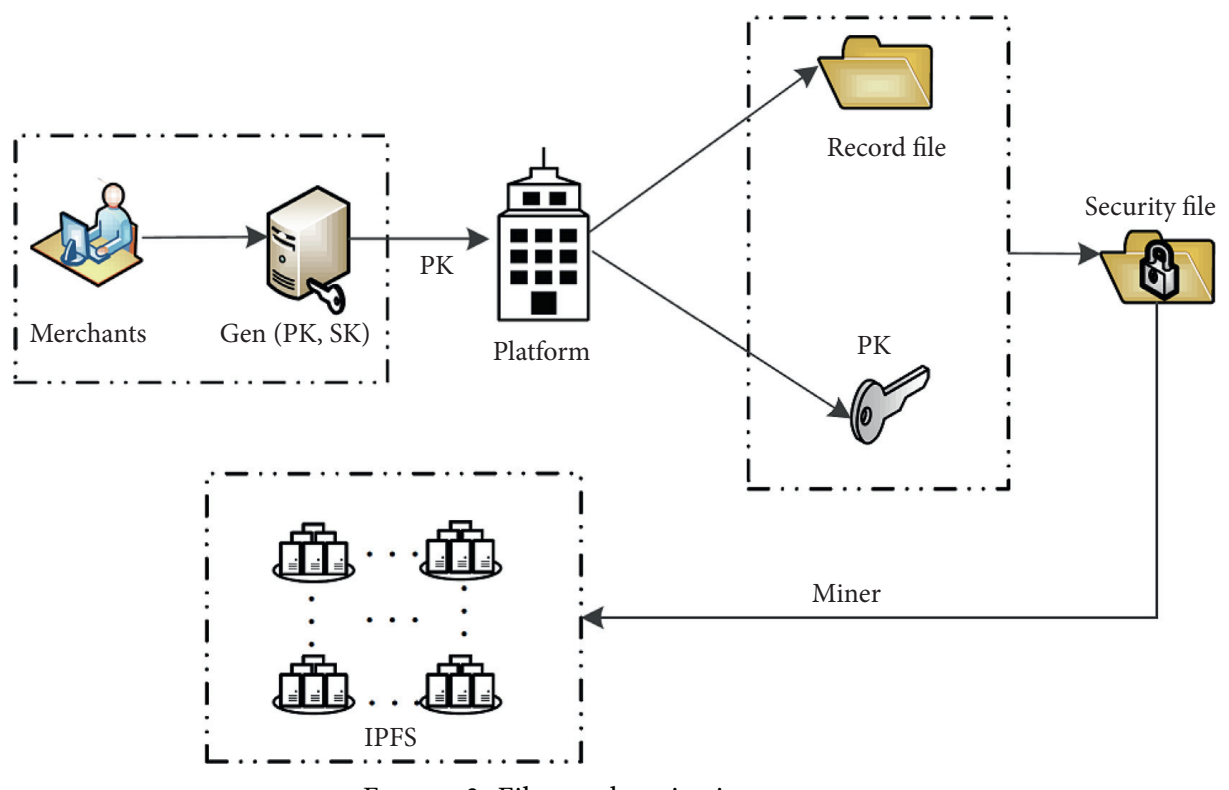

FIgURE 3: File synchronization process.

Input: identity of $j$-th users.

Output: result

(1) Start authentication for $j$-th users

(2) EncrypMi « Encrypt (PKEHRi, uj)

(3) EHR Manager receives encrypted message which is EncryptMi

(4) decrypEHRi « dec (SKEHRi, EncryptMi)

(The message is decrypted by EHR manager with its SK)

EHR Manager sends user uj to Administration Unit

(5) if policy list $(\mathrm{u} j)==$ correct then

(6) Result $\longleftarrow$ ("authentication successful")

(7) Acknowledged user

(8) Else

(9) Result $\longleftarrow$ penalty ( $u j$, action)

(10) End if

Return "Result"

Algorithm 1: User authentication.

Input: Hash of the previous block (PBH), randomly generated list of miners (i.e., Mi).

Output: \{Reception of the block\}

(1) Arrive $M[i]$ from miners that are available

Selected_Miner $[j] \longleftarrow \mathrm{M}[i]$

(2) If Selected_Miner $[j]==$ true then

(3) Build-up MarkelTree(MT)

(4) BlockHeader $\longleftarrow$ (T_stamp $\|\mathrm{MR}\| \mathrm{V} \| \mathrm{PBH})$ (Designed Header of Block)

(5) Compute nonce for the block

(6) BlockHash $\longleftarrow$ (T_stamp $\|\mathrm{MR}\| \mathrm{V}\|\mathrm{PBH}\|$ nonce $)$

(7) If Miner $[j]==$ verified and sig $==$ true and Hash of Block $==$ target-Hash, nonce $=$ correct and Time stamp(TS) $==$ correct then

(8) Result $\longleftarrow$ Block Accepted

(9) Else

(10) Result Block not Accepted

(11) End if

(12) Return: Result

(13) End if 


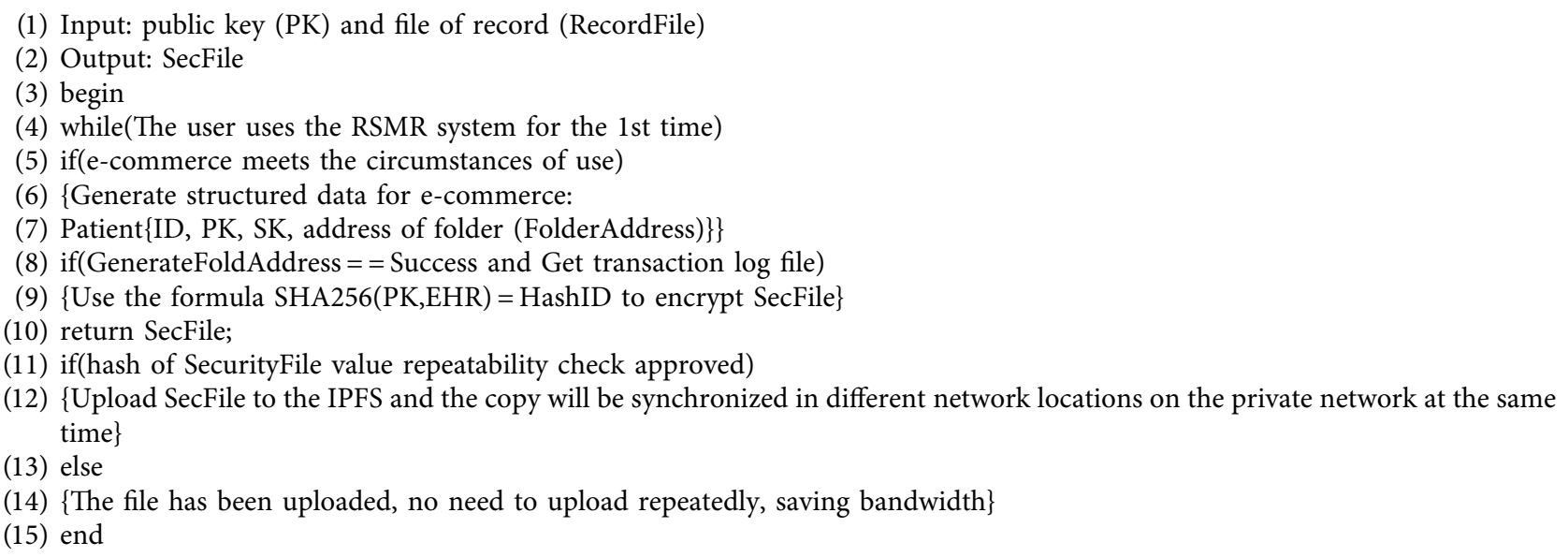

Algorithm 3: File synchronization contract (execution process).

network protocols, we can use the same pair of keys to correspond to different folders. In this paper, we use $\mathrm{EC}_{\mathrm{A}}\left(\mathrm{HashID}_{1}, \ldots, \mathrm{HashID}_{\mathrm{A}}\right)$ to represent the transaction files of different platforms corresponding to the same company.

In the private protocol, we divide the verified and uploaded files into sequences ( sequence $_{1}, \ldots$, sequence ${ }_{n}$ ); in this way, even if the host is shut down or unexpected errors occur, transactions on other platforms will not be affected because we have stored a copy of the file, as shown in Figure 4.

\section{Performance Evaluations}

The performance evaluation of the proposed scheme can be explained as follows.

4.1. Solution Deployment. In the experiment process of this program, five third-generation B-type Raspberry Pis (ARMCortex-A53 1.2 GHz, 1G RAM, Ubuntu OS) are used as hardware carriers to simulate the functions of nodes $\mathrm{A}, \mathrm{B}$, $\mathrm{C}, \mathrm{D}$, and $\mathrm{E}$, and the software program uses the protocol described earlier in this article which is distributed on every hardware carrier. In order to solve the Byzantine problem in the topology, the node management tool IPFS-Cluster is selected for the simulation experiment part of this article, which is divided into the server side and the management side. On the transaction server, the control terminal is typically installed. The aim of this paper is to install the control terminal on point $A$ so that it can handle files and perform the same activities on multiple nodes, such as uploading, upgrading, and file transfer. To execute file transfers, mount the server on $\mathrm{B}, \mathrm{C}, \mathrm{D}$, and $\mathrm{E}$ equipment.

Node object: all participating objects of the trading organization, including platform computers, computers at home for transactions, or a personal PC where transaction records are e-commerce. Model nodes: five nodes, nodes $\mathrm{A}, \mathrm{B}, \mathrm{C}, \mathrm{D}$, and $\mathrm{E}$.
Node A: the computer where the platform wants to synchronize transaction record information

Node B: the computer where the e-commerce company wants to keep personal transaction records

Node C: personal computers of relatives and neighbors related to e-commerce

Nodes D and E: virtual machine image on the platform server

For the smart contract part, this solution uses RemixIDE as the Ethernet smart contract development tool, written in Solidity language and deployed on the Ropsten Test Network and the Ethernet test network.

4.2. Experimental Process. In this paper, we design the following experiments.

4.2.1. Design Key and Synchronize Key. We share all nodes to a public key. If we generate a public key on node a, the smart contract will automatically synchronize to other nodes after execution, which will not affect the node's decryption of the encrypted file, because only the first key generated by point A (node) can decrypt the EMR file.

4.2.2. Start Up the Node. In this paper, we use the cluster management method to take node $\mathrm{A}$ as the starting node. Of course, you can also use other nodes as boot nodes. Its main task is to be responsible for and perform tasks, including public key encryption and uploading files in private protocol and synchronization, so as to form a Blockchain system. The rest of the nodes are copied from the boot node. After the startup, we assign a unique hash ID (qmbxy g9bp), which we use as the single ID of the address of the computer and connect to the 04 host addresses of the other nodes. 


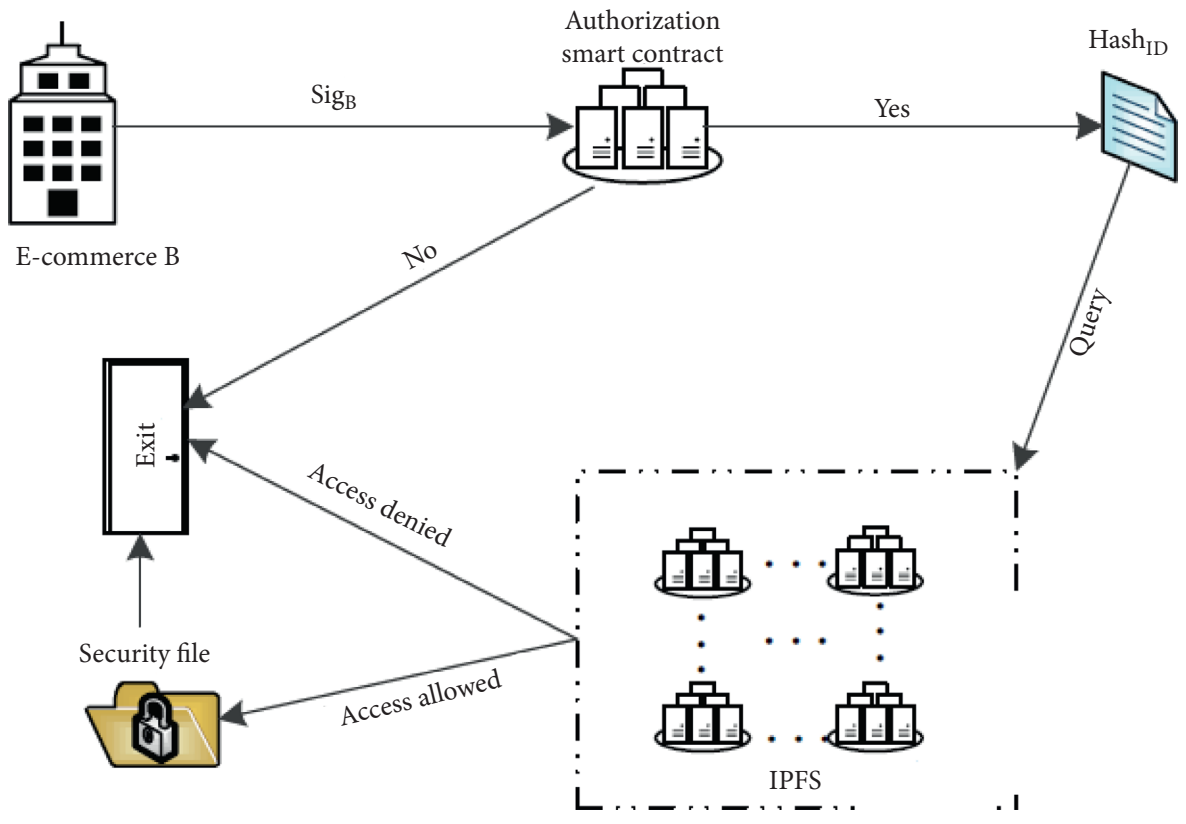

Figure 4: Authorization process.

TABLE 1: Contrast of the features of our scheme with existing schemes.

\begin{tabular}{|c|c|c|c|c|c|c|c|}
\hline S. no. & Feature & {$[15]$} & {$[16]$} & {$[17]$} & {$[18]$} & [19] & Proposed system \\
\hline 1. & Scalability of the system & Yes & No & Yes & Yes & No & Yes \\
\hline 2. & Authentication of users & No & No & Yes & No & No & Yes \\
\hline 3. & Integrity of the system & No & Yes & Yes & No & Yes & Yes \\
\hline 4. & Confidentiality of records & No & Yes & Yes & No & Yes & Yes \\
\hline 5. & Access control mechanism & Yes & Yes & Yes & Yes & Yes & Yes \\
\hline 6. & File synchronization & No & No & No & No & No & Yes \\
\hline 7. & Document traceability & No & No & No & No & No & Yes \\
\hline
\end{tabular}

4.2.3. Upload Files. In Figure 3, we upload the file obtained by using the encryption algorithm. This file only has a unique hash address and upload time. This address is the only address for this article. All identifications are based on this address. Digitize the sequence mentioned above and get an encrypted file.

4.2.4. Transaction Confirmation. When node A uploads to the private protocol network, it can obtain the hash value and time stamp, so that, among all nodes, we only know the upload time and other information, but we cannot see other information in this network. According to the working mechanism of Ethernet, we can also synchronize to the remaining private nodes.

4.2.5. Document Traceability. In the model scenario of this paper, if cross-border business occurs, if e-commerce wants to obtain the files of e-commerce $A$ on the domestic platform, it must obtain the license of the domestic e-commerce platform before downloading and provide the key file. The update of the downloaded transaction information is through the file directory under the management terminal. According to the characteristics of the Blockchain, the file downloads need to synchronize the information of other nodes, so as to realize the file traceability. As shown in Figure 3, when the user enters a hash value, the e-commerce can download the EMR file locally, trigger the algorithm in the smart contract, and then use the private key to decrypt the source file.

The contrast of different features of our proposed scheme with the present schemes is shown in Table 1. From Table 1, it is clear that our proposed system has all the features which the existing schemes have and also has additional features like file synchronization, document traceability, and authentication.

\section{Experiment Analyses}

The intelligent contract part: this program uses Remix-IDE as an Ether Fang Intelligent Contract Development Tool, written in Solidity language, and deploys running on the Etheriquat test network Ropsten Test Network. Experimental hardware and software are shown in Table 2.

Generally speaking, the size of a file is in the range of $1 \sim 5 \mathrm{M}$. In this solution, the upload and download of a file is less than $100 \mathrm{~ms}$. However, in actual application scenarios, an e-commerce platform may usually access hundreds or 
TABLE 2: Hardware and software parameters.

\begin{tabular}{lc}
\hline Software/hardware & Versions \\
\hline Rospberry Pi & $3 \mathrm{~B}$ \\
CPU & Broadcom CortexA53@1.4GHz \\
RAM & 1GB LPDDR2 \\
Operation system & Ubuntu 16.04 LTS \\
IDE & Remix \\
Programming language & Solidity 0.4.7 \\
Compiler environment & JavaScript VM \\
Ethernet & MetaMask \\
Test network & Ropsten Test Network \\
\hline
\end{tabular}

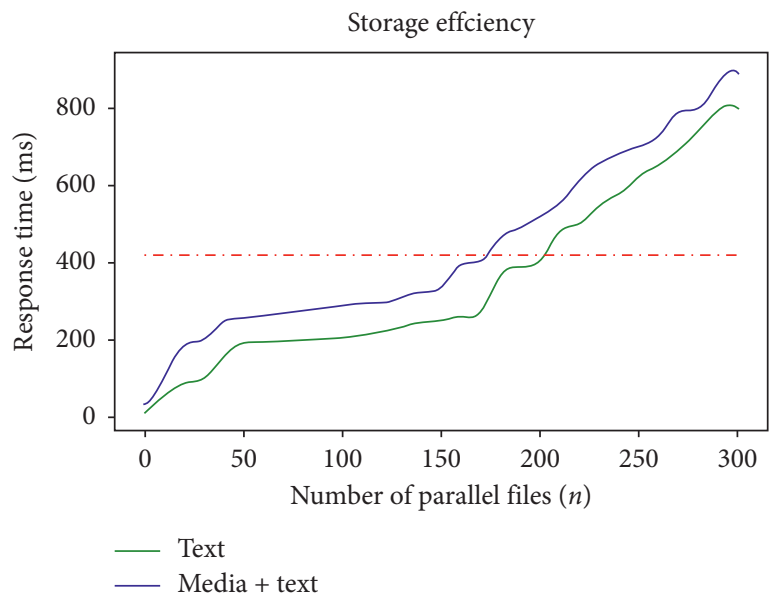

Figure 5: Comparison of storage efficiency.

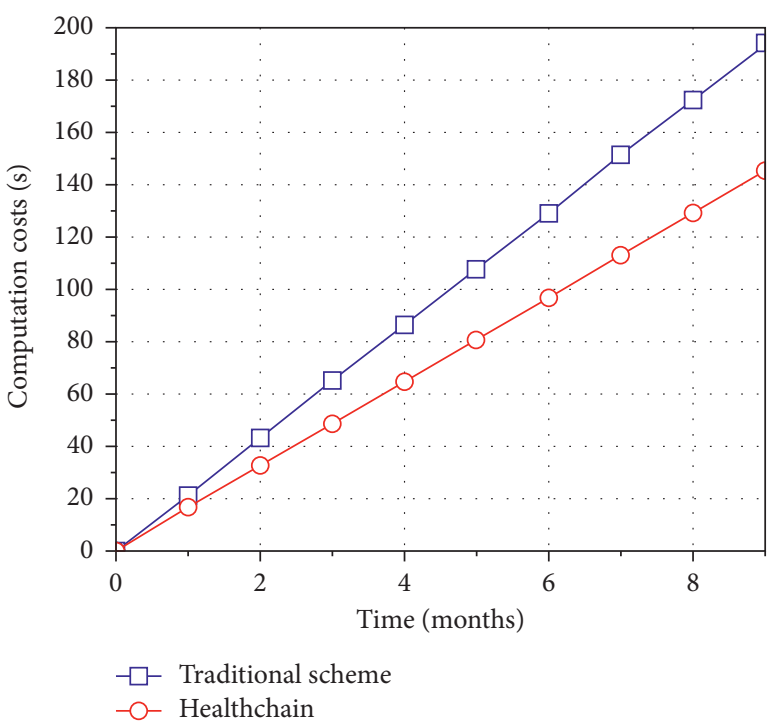

Figure 6: Computation overhead of our proposed system compared to the traditional one.

thousands of transaction files at the same time. Under such high concurrent file access conditions, this experiment simulates parallel transactions from 1 to 300 transaction files. At the same time, considering the actual situation, some transaction files are multimedia files, and some are pure

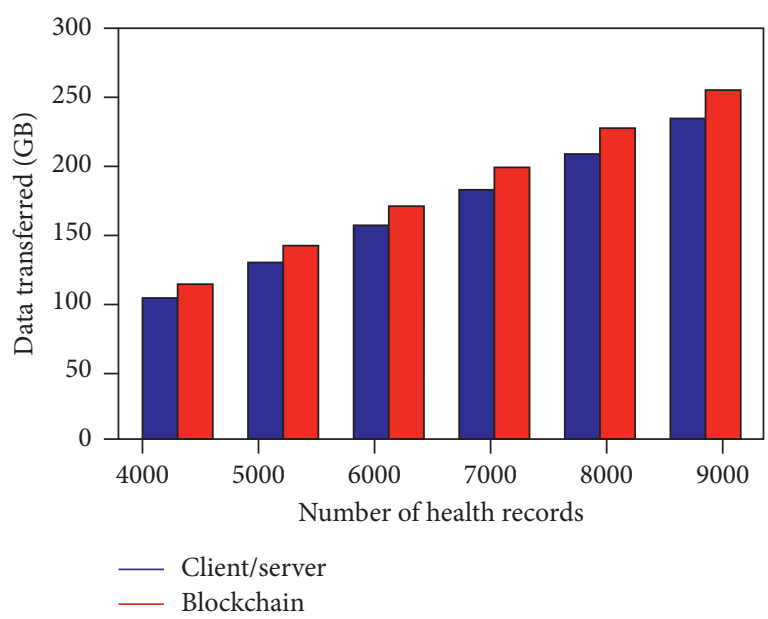

FIgURE 7: Throughput of our proposed system.

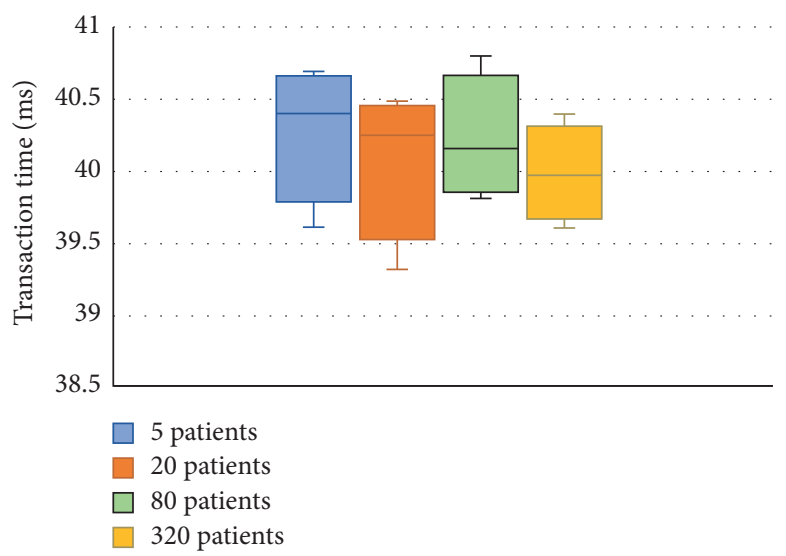

FIgURE 8: The average transaction processing time.

electronic document files. The experimental performance of two different types of files is shown in Figure 5.

It can be seen from Figure 5 that the growth trend of plain text files and multimedia fusion files is basically the same. With the increase of files, the pressure of node confirmation increases and the response time slows down. It can be seen that the response speed of uploading transaction files at the same time is less than 180 files, and the response time is about $400 \mathrm{~ms}$ or less. However, when the number of files is more than 180, the response time has increased rapidly. The possible reason is that, in the experiment based on the POW consensus mechanism, there are too few packaging contract nodes (mining nodes), which leads to slow transactions. In theory, the experiment arranged to the efficiency in the public network will be further optimized. However, in the case of 180 files, the requirements in the model can be fully met, and users will not feel significant delay. Generally speaking, the system performed well without obvious clogging, which was in line with the initial expectations of the study.

The computation overhead of transaction generation of our proposed system and the traditional system is shown in 
Figure 6, the throughput of the proposed system with increasing number of records is shown in Figure 7, and the average transaction of the proposed system per processing time is shown in Figure 8.

\section{Conclusions}

In this research, the application scenario of Blockchain technology in a cross-border e-commerce environment is proposed: cross-domain record sharing and traceability. And the advantages and disadvantages of the proposed modification scheme are analyzed in terms of data protection, access performance, security, implementation difficulty, simplicity of use, and so on. We explained Blockchain and its working according to our work and designed different algorithms which increase the efficiency of our proposed scheme. The experimental results indicate that in spite of success metrics, in the above aspects, the scheme proposed by this research has an effective storage server, low transaction latency, tracking, and reduced power. Though, as multiparty participation and multiparty supervision technology, Blockchain can ensure that transaction records can be traced at any time, but it may also suffer from security issues such as artificial leakage of keys during propagation. In the future, this research will use cryptography such as attribute encryption, homomorphic encryption, and proxy encryption technology, combined with the characteristics of multiparty participation and nontampering in Blockchain technology, to protect user privacy information.

\section{Data Availability}

The data used to support the findings of this study are included within the article.

\section{Conflicts of Interest}

The author declares that there are no conflicts of interest regarding the publication of this study.

\section{References}

[1] S. Nakamoto, "Bitcoin: a peer-to-peer electronic cash system," 2008, https://bitcoin.org/en/bitcoin-paper.

[2] V. Buterin, "A next-generation smart contract and decentralized application platform," 2014, https:/github.com/ ethereum/wiki/wiki/White-Paper.

[3] X. Yu, Y. Chu, F. Jiang, Y. Guo, and D. Gong, "SVMs classification based two-side cross domain collaborative filtering by inferring intrinsic user and item features," KnowledgeBased Systems, vol. 141, pp. 80-91, 2018.

[4] UK Government Chief Scientific Adviser, "Distributed Ledger technology: beyond Blockchain," 2016, https://assets. publishing.service.gov.uk/government/uploads/system/uploads/ attachment_data/file/492972/gs-16-1-distributed-ledgertechnology.pdf.

[5] X. Yu, Q. Hu, H. Li, J. Du, J. Gao, and L. Sun, "Cross-domain recommendation based on latent factor alignment," Neural Computing and Applications, vol. 14, 2021.
[6] Y. Yuan and F. Wang, "Blockchain: the state of the art and future trends," Acta Automatica Sinica, vol. 42, no. 4, pp. 481-494, 2016.

[7] J. Frizzo-Barker, P. A. Chow-White, P. R. Adams, J. Mentanko, D. Ha, and S. Green, "Blockchain as a disruptive technology for business: a systematic review," International Journal of Information Management, vol. 51, 2020.

[8] M. Usman, M. A. Jan, and A. Jolfaei, "SPEED: a deep learning assisted privacy-preserved framework for intelligent transportation systems," IEEE Transactions on Intelligent Transportation Systems, vol. 3, pp. 1-9, 2020.

[9] R. Cole, M. Stevenson, and J. Aitken, "Blockchain technology: implications for operations and supply chain management," Supply Chain Management: An International Journal, vol. 24, no. 4, pp. 469-483, 2019.

[10] N. Kshetri, "1 Blockchain's roles in meeting key supply chain management objectives," International Journal of Information Management, vol. 39, pp. 80-89, 2018.

[11] M. A. Jan, J. Cai, X. C. Gao et al., "Security and Blockchain convergence with Internet of Multimedia Things: current trends, research challenges and future directions," Journal of Network and Computer Applications, vol. 175, pp. 102918102935, 2021.

[12] Y. Chang, E. Iakovou, and W. Shi, "Blockchain in global supply chains and cross border trade: a critical synthesis of the state-of-the-art, challenges and opportunities," International Journal of Production Research, vol. 58, no. 7, pp. 2082-2099, 2019.

[13] S. Fosso Wamba, A. Gunasekaran, R. Dubey, and E. W. T. Ngai, "Big data analytics in operations and supply chain management," Annals of Operations Research, vol. 270, no. 1-2, pp. 1-4, 2018.

[14] L.-W. Wong, L.-Y. Leong, J.-J. Hew, G. W.-H. Tan, and K.-B. Ooi, "Time to seize the digital evolution: adoption of Blockchain in operations and supply chain management among Malaysian SMEs," International Journal of Information Management, vol. 52, 2020.

[15] P. Zhang, M. A. Walker, J. White, D. C. Schmidt, and G. Lenz, "Metrics for assessing Blockchain-based healthcare decentralized apps," in Proceedings of the 2017 IEEE 19th International Conference One-Health Networking, Applications and Services (Healthcom), pp. 1-4, Dalian, China, October 2017.

[16] A. Azaria, A. Ekblaw, T. Vieira, and A. Lippman, "Medrec: using Blockchain for medical data access and permissionmanagement," in Proceedings of the 2016 2nd International Conference on Openand Big Data (OBD), pp. 25-30, Vienna, Austria, August 2016.

[17] Q. Xia, E. B. Sifah, K. O. Asamoah, J. Gao, X. Du, and M. Guizani, "Medshare: trust-less medical data sharing among cloud service providers via Blockchain," IEEE Access, vol. 5, pp. 14757-14767, 2017.

[18] Q. Xia, E. Sifah, A. Smahi, S. Amofa, and X. Zhang, "Bbds: Blockchain-based data sharing for electronic medical records in cloud environments," Information, vol. 8, no. 2, p. 44, 2017.

[19] M. A. Jan, F. Khan, R. Khan et al., "A lightweight mutual authentication and privacy-preservation scheme for intelligent wearable devices in industrial-CPS," IEEE Transactions on Industrial Informatics, vol. 17, no. 8, pp. 5829-5839, 2021.

[20] D. Wang and Z. Xie, "Application path and legal regulatory framework of Blockchain technology in the collaborative development of cross-border e-commerce," Journal of Xinjiang University of Finance and Economics, vol. 1, no. 3, pp. 64-71, 2020. 
[21] J. Wang, W. Zhao, R. Gao, and J. Cao, "Research on the application of Blockchain technology in cross border e-commerce," Jilin Financial Research, vol. 3, no. 8, pp. 11-13 $+30,2020$.

[22] L. Liu, D. Cheng, and X. Su, "Analysis of consumer trust mechanism based on Blockchain technology," Commercial Economy Research, vol. 11, no. 15, pp. 32-36, 2020.

[23] Z. Jin, "Application of Blockchain technology in e-commerce logistics industry," Electronic Technology and Software Engineering, vol. 6, no. 15, pp. 175-176, 2020.

[24] C. Qian, "Analysis on the financial management of e-commerce enterprises based on "Blockchain + Internet"," Journal of Times Finance, vol. 4, no. 21, pp. 79-80, 2020.

[25] M. Wu and Y. Liu, "Research on the construction of crossborder payment system driven by Blockchain technologyfrom the perspective of cost control," Journal of Guangdong Polytechnic of Light Industry, vol. 19, no. 2, pp. 14-18, 2020.

[26] H. Jia, "Application of "Blockchain + e-commerce" in the field of e-commerce of agricultural products in Weinan City," Computer Technology and Development, vol. 30, no. 6, pp. 191-196, 2020.

[27] J. Pang and H. Huize, "Analysis and supervision of crossborder payment model based on Blockchain technology," Journal of Economist, vol. 6, no. 6, pp. 56-57, 2020.

[28] X. Chen, "Optimization of financial activities of cross border e-commerce enterprises based on Blockchain technology," Commercial Economy, vol. 7, no. 5, pp. 146-148, 2020.

[29] X. Jiang, Research on the Application of Blockchain in Cross Border E-Commerce, Tianjin University of Commerce, Tianjin, China, 2020.

[30] S. Yang, Z. Huang, and D. Li, "Application scenarios and value analysis of the Blockchain in cross border e-commerce," Cyberspace Security, vol. 11, no. 4, pp. 44-48, 2020.

[31] Y. Liu and L. Qiu, "Solving mechanism of "trust" gap of crossborder e-commerce under Blockchain technology," Commercial Economy Research, vol. 10, no. 7, pp. 150-153, 2020.

[32] Y. Yi, "Prospects for the development of cross border logistics finance in China based on Blockchain technology-taking accompanying payment as an example," Foreign Trade Practice, vol. 11, no. 4, pp. 77-80, 2020.

[33] D. Yan and F. Zhu, "Research on breaking the credit crisis of China's cross-border e-commerce under the background of Blockchain," Time-Honored Brand Marketing, vol. 6, no. 4, pp. 18-20, 2020.

[34] M. Yin and X. Xu, "Challenges faced by cross-border e-commerce export trade and Countermeasures: from the perspective of Blockchain technology application," Commercial Economy Research, vol. 2, no. 6, pp. 149-152, 2020.

[35] Q. Yi, H. Yang, and K. Wang, "Review on the application of Blockchain technology in logistics," Logistics Technology, vol. 39, no. 3, pp. 129-133, 2020.

[36] X. Yu, J. Yang, and Z. Xie, "Training SVMs on a bound vectors set based on Fisher projection," Frontiers of Computer Science, vol. 8, no. 5, pp. 793-806, 2014.

[37] X. Yu, F. Jiang, J. Du, and D. Gong, “A cross-domain collaborative filtering algorithm with expanding user and item features via the latent factor space of auxiliary domains," Pattern Recognition, vol. 94, pp. 96-109, 2019.

[38] J. Ge, S. Jiao, and C. Weng, "Development opportunities and challenges of "Blockchain + cross border e-commerce"," Modern Economic Information, vol. 8, no. 24, pp. 310-311, 2019. 